\title{
An Explicit Representation of the Remainder of some Newton-Cotes Formulas in Terms of Higher Order Differences
}

\author{
B. BUtTGENBACH, G. LUtTGens and R. J. Nessel
}

Depending upon the exactness of the rule, the remainders of some Newton-Cotes formulas are explicitly represented in terms of higher order differences. Consequently, those error bounds for the associated compound quadrature processes, given via corresponding moduli of continuity, may now be established in a completely elementary way, in fact with good constants. As an application of previous quantitative extensions of the uniform boundedness principle it is finally shown that the error estimates considered are always sharp.

Key words: error representations; midpoint, trapezoidal, Simpson, 3/8-, Milne rule; sharpness of error bounds

AMS subject classification: 41 A 25, 41 A 55, 65 D 32

Let $C[a, b]$ be the Banach space of functions $f$, continuous on the compact interval $[a, b]$ of the real axis $\mathbf{R}$, endowed with the usual norm $\|f\|_{C}=\max \{|f(u)|: u \in[a, b]\}$. Given $f \in C[0,1]$, consider the (elementary) midpoint rule $Q^{M i} f=f(1 / 2)$ for the approximate calculation of the integral $I f=\int_{0}^{1} f(u) d u$. For the remainder $R^{M i}=Q^{M i}-I$ there obviously holds true the representation

$$
\begin{aligned}
R^{M_{i}} f & =f(1 / 2)-\int_{0}^{1} f(u) d u \\
& =\int_{0}^{1 / 2}\left[-f\left(\frac{1}{2}-u\right)+2 f\left(\frac{1}{2}\right)-f\left(\frac{1}{2}+u\right)\right] d u=\int_{0}^{1 / 2}\left[-\Delta_{h}^{2} f\left(\frac{1}{2}-h\right)\right] d h,
\end{aligned}
$$

where $\Delta_{h}^{r} f(x)=\Delta_{h}\left(\Delta_{h}^{r-1} f(x)\right), \Delta_{h} f(x)=f(x+h)-f(x), r \in \mathrm{N}$ (= set of natural numbers) denotes the r-th difference with increment $h \in \mathrm{R}$. Note that (1) precisely corresponds to the familiar fact that $Q^{M i}$ is exact for polynomials of degree 1 . Representations of a similar nature for the trapezoidal rule $Q^{\operatorname{Tr}} f=[f(0)+f(1)] / 2$ were already employed by several authors (cf. $[5 ; 6 ; 7$, pp. 43,54$]$ ), too. The following version turns out to be particularly suitable for extensions to formulas of higher order:

$$
\begin{aligned}
R^{T r} f & =\frac{1}{2}[f(0)+f(1)]-\int_{0}^{1} f(u) d u \\
& =\int_{0}^{1 / 2}([f(0)-2 f(u)+f(2 u)]+[f(1)-2 f(1-u)+f(1-2 u)]) d u \\
& =\int_{0}^{1 / 2}\left[\Delta_{h}^{2} f(0)+\Delta_{-h}^{2} f(1)\right] d h .
\end{aligned}
$$

Indeed, in view of the identities (1), (2) one may now expect that for formulas which are exact on $\mathcal{P}_{r-1}(=$ set of algebraic polynomials of degrec $(r-1)$ ) there hold true corresponding representations for the remainders in terms of appropriate $r$-th differences. The following 
confirms this for the Simpson, 3/8-, and Milne rule, i.e., for

$$
\begin{aligned}
Q^{S_{i}} f & =\frac{1}{6}\left[f(0)+4 f\left(\frac{1}{2}\right)+f(1)\right] \\
Q^{3 / 8} f & =\frac{1}{8}\left[f(0)+3 f\left(\frac{1}{3}\right)+3 f\left(\frac{2}{3}\right)+f(1)\right], \\
Q^{M i l} f & =\frac{1}{90}\left[7 f(0)+32 f\left(\frac{1}{4}\right)+12 f\left(\frac{1}{2}\right)+32 f\left(\frac{3}{4}\right)+7 f(1)\right] .
\end{aligned}
$$

Theorem 1: For $f \in C[0,1]$ and the corresponding remainders $R^{\circ} f=Q^{\circ} f-\int_{0}^{1} f(u) d u$ one has the representations

$$
\begin{aligned}
R^{S i} f= & \frac{2}{3} \int_{0}^{1 / 4}\left[\Delta_{h}^{4} f(0)+\frac{2}{3} \Delta_{h}^{4} f\left(\frac{1}{2}-2 h\right)+\Delta_{-h}^{4} f(1)\right] d h, \\
R^{3 / 8} f= & \frac{3}{4} \int_{0}^{1 / 6}\left[\Delta_{h}^{4} f(0)+3 \Delta_{h}^{4} f\left(\frac{1}{3}\right)+3 \Delta_{-h}^{4} f\left(\frac{2}{3}\right)+\Delta_{-h}^{4} f(1)\right] d h, \\
R^{M i l} f= & \int_{0}^{1 / 8}\left(\frac{28}{45}\left[\Delta_{h}^{6} f(0)+\Delta_{-h}^{6} f(1)\right]+\frac{232}{75}\left[\Delta_{h}^{6} f\left(\frac{1}{4}\right)+\Delta_{-h}^{6} f\left(\frac{3}{4}\right)\right]\right. \\
& \left.+\frac{28}{675}\left[\Delta_{h}^{6} f\left(\frac{1}{4}-h\right)+\Delta_{-h}^{6} f\left(\frac{3}{4}+h\right)\right]+\frac{8}{225}\left[\Delta_{h}^{6} f\left(\frac{1}{2}-2 h\right)+\Delta_{-h}^{6} f\left(\frac{1}{2}+2 h\right)\right]\right) d h .
\end{aligned}
$$

Proof: Once a candidate is available, the proof may proceed by verification. Thus for the Simpson rule

$$
\begin{aligned}
& \int_{0}^{1 / 4}([f(0)-4 f(h)+6 f(2 h)-4 f(3 h)+f(4 h)] \\
& \quad+\frac{2}{3}\left[f\left(\frac{1}{2}-2 h\right)-4 f\left(\frac{1}{2}-h\right)+6 f\left(\frac{1}{2}\right)-4 f\left(\frac{1}{2}+h\right)+f\left(\frac{1}{2}+2 h\right)\right] \\
& +[f(1)-4 f(1-h)+6 f(1-2 h)-4 f(1-3 h)+f(1-4 h)]) d h \\
& =\frac{1}{4} f(0)+\left(-4 \int_{0}^{1 / 4}+3 \int_{0}^{2 / 4}-\frac{4}{3} \int_{0}^{3 / 4}+\frac{1}{4} \int_{0}^{1}\right) f(u) d u \\
& +\frac{2}{3}\left[\frac{6}{4} f\left(\frac{1}{2}\right)+\left(\frac{1}{2} \int_{0}^{1 / 2}-4 \int_{1 / 4}^{1 / 2}-4 \int_{1 / 2}^{3 / 4}+\frac{1}{2} \int_{1 / 2}^{1}\right) f(u) d u\right] \\
& +\frac{1}{4} f(1)+\left(-4 \int_{3 / 4}^{1}+3 \int_{2 / 4}^{1}-\frac{4}{3} \int_{1 / 4}^{1}+\frac{1}{4} \int_{0}^{1}\right) f(u) d u \\
& =\frac{1}{4} f(0)+f\left(\frac{1}{2}\right)+\frac{1}{4} f(1)-\frac{3}{2} \int_{0}^{1} f(u) d u=\frac{3}{2} R^{s i} f .
\end{aligned}
$$

To indicate how to develop an actual candidate, let us try a representation of $R^{3 / 8} f$ via

$$
\int_{0}^{1 / 6}\left[b_{1} \Delta_{h}^{4} f(0)+b_{2} \Delta_{h}^{4} f\left(\frac{1}{3}\right)+b_{3} \Delta_{-h}^{4} f\left(\frac{2}{3}\right)+b_{4} \Delta_{-h}^{4} f(1)\right] d h,
$$

thus one considers a fourth difference $\Delta_{ \pm h}^{4}$ (the rule being exact on $\mathcal{P}_{3}$ ) at each knot, the interval of integration being again a half of the distance of the knots. Comparing coefficients 
at $f(j / 3)$ delivers $b_{1}=b_{4}=3 / 4, b_{2}=b_{3}=9 / 4$, and in fact the remaining integrals then fit together to $-\int_{0}^{1} f(u) d u$. Unfortunately, this reasoning for $R^{3 / 8}$ does not suggest a general procedure. Indeed, the candidate for $R^{M i l}$ has to be chosen even more complicated ( 8 differences instead of 5). The correctness of the representation for $R^{M i l}$ follows by verification.

Of course it would be interesting to have a general procedure to establish representations like those of Theorem 1 for a wide class of (elementary) quadrature formulas

$$
R^{e l} f=Q^{e l} f-I f=\sum_{i=1}^{j} a_{i} f\left(x_{i}\right)-\int_{0}^{1} f(u) d u,
$$

where $0 \leq x_{1}<\ldots<x_{j} \leq 1$ and the formula is assumed to be exact on $\mathcal{P}_{r-1}$. But so far we have to leave this as an open problem.

Nevertheless, the rules (1-5), though particular, generate those compound quadrature processes, most commonly used in the applications. In this connection the next result shows how a representation for the remainder (6) of an elementary quadrature rule $Q^{\text {el }}$ transfers to the rule

$$
R^{[a, b]} f=Q^{[a, b]} f-I f=(b-a) \sum_{i=1}^{j} a_{i} f\left(a+(b-a) x_{i}\right)-\int_{a}^{b} f(u) d u,
$$

obtained by an affine transformation of $[0,1]$ to the interval $[a, b]$, or to the compound quadrature process

$$
R_{(n)} f=Q_{(n)} f-I f=\frac{b-a}{n} \sum_{k=1}^{n} \sum_{i=1}^{j} a_{i} f\left(a+\left(k-1+x_{i}\right) \frac{b-a}{n}\right)-\int_{a}^{b} f(u) d u,
$$

generated by the elementary rule (6).

Theorem 2: Suppose that for the remainder (6) of an elementary rule there holds true the representation

$$
R^{e l} f=\sum_{l=1}^{m} \int_{0}^{s} b_{l} \Delta_{h}^{r} f\left(y_{l}+c_{l} h\right) d h
$$

for some $m, r \in \mathrm{N}, 0<s<1, c_{l} \in \mathbf{R}$, and $y_{l} \in\left\{x_{i}: 1 \leq i \leq j\right\}$. Then one has for $f \in C[a, b]$

$$
\begin{aligned}
& R^{[a, b]} f=\sum_{l=1}^{m} \int_{0}^{s(b-a)} b_{l} \Delta_{h}^{r} f\left(a+(b-a) y_{l}+c_{l} h\right) d h, \\
& R_{(n)} f=\sum_{k=1}^{n} \sum_{l=1}^{m} \int_{0}^{s(b-a) / n} b_{l} \Delta_{h} f\left(a+\left(k-1+y_{l}\right) \frac{b-a}{n}+c_{l} h\right) d h .
\end{aligned}
$$

Proof: Setting $g(x)=f(a+(b-a) x)$ one has

$$
\begin{aligned}
R^{[a, b]} f & =(b-a)\left[\sum_{i=1}^{j} a_{i} g\left(x_{i}\right)-\int_{0}^{1} g(u) d u\right]=(b-a) R^{e l} g \\
& =(b-a) \sum_{l=1}^{m} \int_{0}^{s} b_{l} \Delta_{h}^{r} g\left(y_{l}+c_{l} h\right) d h \\
& =(b-a) \sum_{l=1}^{m} \int_{0}^{s} b_{l} \sum_{k=0}^{r}(-1)^{r-k}\left(\begin{array}{l}
r \\
k
\end{array}\right) f\left(a+(b-a)\left[y_{l}+c_{l} h+k h\right]\right) d h
\end{aligned}
$$


which establishes the representation for $R^{[a, b]} f$. This in turn implies

$$
\begin{aligned}
R_{(n)} f & =\sum_{k=1}^{n} R^{[a+(k-1)(b-a) / n, a+k(b-a) / n]} f \\
& =\sum_{k=1}^{n} \sum_{l=1}^{m} \int_{0}^{s(b-a) / n} b_{l} \Delta_{h}^{r} f\left(a+(k-1) \frac{b-a}{n}+\frac{b-a}{n} y_{l}+c_{l} h\right) d h .
\end{aligned}
$$

Observing that any backward difference $\Delta_{-h}$ may also be rewritten as a forward one $\Delta_{h}$ it is obvious that those representations previously established are all of the form (9) (note that $y_{l_{1}}=y_{l_{2}}$ for $l_{1} \neq l_{2}$ is possible).

Once representations like those of Theorem 1 are available, estimates of the remainders in terms of the $r$-th modulus of continuity of $f$

$$
\omega_{r}(\delta, f,[a, b])=\sup \left\{\left|\Delta_{h}^{r} f(x)\right|: x, x+r h \in[a, b],|h| \leq \delta\right\}
$$

are immediate. In fact, it turns out that the constants resulting are rather good. For example, for the elementary rules (1)-(5) one has

Corollary: For $f \in C[0,1]$ there holds true

$$
\begin{aligned}
\left|R^{M i} f\right| & \leq \frac{1}{2} \omega_{2}\left(\frac{1}{2}, f,[0,1]\right), & & \left|R^{T r} f\right| \leq \omega_{2}\left(\frac{1}{2}, f,[0,1]\right), \\
\left|R^{S i} f\right| & \leq \frac{4}{9} \omega_{4}\left(\frac{1}{4}, f,[0,1]\right), & & \left|R^{3 / 8} f\right| \leq \omega_{4}\left(\frac{1}{6}, f,[0,1]\right), \\
\left|R^{M i l} f\right| & \leq \frac{128}{135} \omega_{6}\left(\frac{1}{8}, f,[0,1]\right) . & &
\end{aligned}
$$

From a good estimate for the elementary rule (6) one immediately obtains a corresponding one for the affine transformation (7) and the compound process (8). Indeed,

Theorem 3: Given the elementary rule (6), suppose that there holds true the estimate

$$
\left|R^{e l} g\right| \leq c \omega_{r}(\delta, g,[0,1]) \quad(g \in C[0,1]) .
$$

Then one has for $f \in C[a, b]$

$$
\begin{aligned}
\left|R^{[a, b]} f\right| & \leq c(b-a) \omega_{r}(\delta(b-a), f,[a, b]) \\
\left|R_{(n)} f\right| & \leq c(b-a) \omega_{r}\left(\delta \frac{b-a}{n}, f,[a, b]\right) .
\end{aligned}
$$

Proof: With the substitution $g(x)=f(a+x(b-a))$ it again follows that

$$
\left|R^{[a, b]} f\right|=(b-a)\left|R^{e l} g\right| \leq c(b-a) \omega_{r}(\delta, g,[0,1]) \leq c(b-a) \omega_{r}(\delta(b-a), f,[a, b]) .
$$

This in turn implies

$$
\left|R_{(n)} f\right| \leq \sum_{k=1}^{n}\left|R^{[a+(k-1)(b-a) / n, a+k(b-a) / n]} f\right| \leq \sum_{k=1}^{n} c \frac{b-a}{n} \omega_{r}\left(\delta \frac{b-a}{n}, f,[a, b]\right) .
$$


For example, for the compound midpoint, trapezoidal, and Simpson rule one has that for every $f \in C[a, b]$ (cf. Corollary)

$$
\begin{aligned}
& \left|R_{(n)}^{M i} f\right| \leq \frac{b-a}{2} \omega_{2}\left(\frac{b-a}{2 n}, f,[a, b]\right), \\
& \left|R_{(n)}^{T r} f\right| \leq(b-a) \omega_{2}\left(\frac{b-a}{2 n}, f,[a, b]\right), \\
& \left|R_{(n)}^{S i} f\right| \leq \frac{4(b-a)}{9} \omega_{4}\left(\frac{b-a}{4 n}, f,[a, b]\right),
\end{aligned}
$$

respectively. Estimates of this type, but with unspecified constants, are well known. Usually (cf. [1]) one proceeds via an interpolation argument, thus employs the exactness of the rule and Peano's theorem to derive an estimate for smooth functions which together with the boundedness of the process delivers an estimate versus a corresponding K-functional. The rather intricate equivalence (with unspecified constants) between this K-functional and $\omega_{r}$ establishes the estimate for general processes. In contrast, our approach is completely elementary, but so far only works for the particular rules (1)-(5), at the same time, however, delivering good constants. In this connection it may be worthwhile mentioning that in [7, pp. $41 ; 43,54]$ an elementary approach (like the one stressed here) is employed to establish (10), (11), but for the Simpson rule an interpolation argument as described above is used. Indeed, since the problem of best constants is raised in [7] at several places (cf. [7, pp. 52, $60,64]$ ), this may also be considered as a motivation to work out an elementary approach for the Simpson rule, too.

Let us conclude with the observation that it may generally be shown that estimates of type (10)-(12) are always sharp (with regard to the order) for compound quadrature processes. If the modulus of continuity $\omega_{r}(\delta, f)$ of the function $f$ behaves like $\delta^{r}$, this follows from familiar asymptotic expansions of the remainder or by testing the counterexample $x^{r}$. In all the other cases one may proceed via the following quantitative extension of the uniform boundedness principle: For a Banach space $X$ (with norm $\|\cdot\|$ ) let $X^{*}$ be the set of sublinear, bounded functionals $T$ on $X$, i.e., $T$ maps $X$ into $\mathrm{R}$ such that for all $f, g \in X, \alpha \in \mathbf{R}$

$$
\begin{gathered}
|T(f+g)| \leq|T f|+|T g|, \quad|T(\alpha f)|=|\alpha||T f|, \\
\|T\|_{X} \cdot=\sup \{|T f|:\|f\| \leq 1\}<\infty .
\end{gathered}
$$

Let $\omega$ be an abstract modulus of continuity, thus a function, continuous on $[0, \infty)$ with

$$
0=\omega(0)<\omega(s) \leq \omega(s+t) \leq \omega(s)+\omega(t) \quad(s, t>0),
$$

additionally satisfying

$$
\lim _{t \rightarrow 0^{+}} \frac{\omega(t)}{t}=\infty
$$

(e.g., $\omega(t)=t^{\alpha}$ for $0<\alpha<1$ ). Let $\sigma(t)$ be a function, (strictly) positive on $(0, \infty)$, and $\left\{\varphi_{n}\right\} \subset \mathbf{R}$ be a sequence, (strictly) decreasing with $\lim _{n \rightarrow \infty} \varphi_{n}=0$. In these terms one has

Theorem 4: Suppose that for the remainder $\left\{T_{n}: n \in \mathrm{N}\right\} \subset X^{*}$ of some approximation process and for a measure of smoothness $\left\{U_{t}: t>0\right\} \subset X^{*}$ there are test elements $g_{n} \in X$ with $(t \rightarrow 0+, n \rightarrow \infty)$

$$
\begin{aligned}
\left\|g_{n}\right\| & =\mathcal{O}(1) \\
T_{n} g_{n} & \neq \mathcal{O}(1) \\
\left|U_{t} g_{n}\right| & \leq C \min \left\{1, \sigma(t) / \varphi_{n}\right\} \quad(t>0, n \in \mathrm{N})
\end{aligned}
$$


Then for each modulus $\omega$ satisfying (13), (14) there exists a counterexample $f_{\omega} \in X$ with

$$
\begin{aligned}
\left|U_{t} f_{\omega}\right| & =\mathcal{O}(\omega(\sigma(t))), \\
T_{n} f_{\omega} & \neq \mathcal{O}\left(\omega\left(\varphi_{n}\right)\right) .
\end{aligned}
$$

For a proof as well as for a number of applications, explicitly worked out, sec [2-4] (and the literature cited there). Here we would like to apply Theorem 4 to compound quadrature processes.

Theorem 5: Given the compound quadrature process (8), suppose that there holds true the estimate

$$
\left|R_{(n)} \int\right| \leq C \omega_{r}\left(\frac{1}{n}, f,[a, b]\right) \quad(f \in C[a, b]) .
$$

Then this estimate is sharp in the sense that for each modulus $\omega$ satisfying (13), (14) there exists a counterexample $f_{\omega} \in C[a, b]$ with

$$
\omega_{r}\left(t, f_{\omega},[a, b]\right)=\mathcal{O}\left(\omega\left(t^{r}\right)\right) \quad(t \rightarrow 0+),
$$

thus $\left|R_{(n)} f_{w}\right|=\mathcal{O}\left(\omega\left(n^{-r}\right)\right)$, but on the other hand

$$
R_{(n)} f_{\omega} \neq \mathcal{O}\left(\omega\left(n^{-r}\right)\right) \quad(n \rightarrow \infty) .
$$

Proof: To apply Theorem 4, set $X=C[a, b], \varphi_{n}=n^{-r}, \sigma(t)=t^{r}, T_{n}=R_{(n)}, U_{t} f=$ $\omega_{r}(t, f,[a, b]) \in(C[a, b])^{*}$, and (cf. (6), (8))

$$
g_{n}(x)=\prod_{i=1}^{j} \sin ^{2}\left(-\frac{a \pi n}{b-a}-\pi x_{i}+\frac{\pi n}{b-a} x\right) \in C[a, b] .
$$

Obviously, $\left\|g_{n}\right\|_{C} \leq 1$, thus (15). Since the testelement $g_{n}$ is chosen such that it vanishes at the knots of the rule $Q_{(n)}$, one has $Q_{(n)} g_{n}=0$, and therefore

$$
\begin{aligned}
\left|R_{(n)} g_{n}\right| & =\int_{a}^{b} g_{n}(u) d u=\frac{b-a}{\pi n} \int_{0}^{\pi n} \prod_{i=1}^{j} \sin ^{2}\left(-\pi x_{i}+u\right) d u \\
& =\frac{b-a}{\pi n} \sum_{k=0}^{n-1} \int_{k \pi}^{(k+1) \pi} \prod_{i=1}^{j} \sin ^{2}\left(-\pi x_{i}+u\right) d u=\frac{b-a}{\pi} \int_{0}^{\pi} \prod_{i=1}^{j} \sin ^{2}\left(-\pi x_{i}+v\right) d v,
\end{aligned}
$$

thus (16). Concerning (17), on the one hand $\omega_{r}\left(t, g_{n}\right) \leq 2^{r}\left\|g_{n}\right\|_{C} \leq 2^{r}$, on the other hand, since

$$
g_{n}(x)=2^{-j} \prod_{i=1}^{j}\left[1-\cos \left(-2 \frac{a \pi n}{b-a}-2 \pi x_{i}+2 \frac{\pi n}{b-a} x\right)\right]=A_{o}+\sum_{k=1}^{M} A_{k} \cos \left(d_{k}+e_{k} n x\right)
$$

for some constants $A_{k}, e_{k}, M$, independent of $n$, it also follows that

$$
\begin{aligned}
\omega_{r}\left(t, g_{n},[a, b]\right) & \leq \sum_{k=1}^{M}\left|A_{k}\right| \omega_{r}\left(t, \cos \left(d_{k}+e_{k} n x\right),[a, b]\right) \\
& \leq \sum_{k=1}^{M}\left|A_{k}\right| t^{r}\left\|\left(\frac{d}{d x}\right)^{r} \cos \left(d_{k}+e_{k} n x\right)\right\|_{C} \leq \sum_{k=1}^{M}\left|A_{k}\right| t^{r}\left|e_{k}\right|^{r} n^{r}
\end{aligned}
$$


Now the assertions are an immediate consequence of Theorem 4.

For the quadrature rules (1)-(5), mainly considered in this note, the direct result (18) is established by Theorem 3 (and the Corollary). On the other hand, it is well known that (18) holds true for arbitrary compound quadrature processes if the generating elementary rule is exact on $\mathcal{P}_{r-1}$. Indeed, using Peano's theorem one immediately deduces an estimate versus the corresponding $K$-functional, and its equivalence to $\omega_{r}$ completes the argument.

\section{REFERENCES}

[1] BUTZER,P.L., SCHERER, K. and U. WESTPHAL: On the Banach-Steinhaus theorem and approximation in Jocally convex spaces. Acta Sci. Math.(Szeged) 34 (1973), $25-34$.

[2] DICKMEIS, W. and R.J. NESSEL: On uniform boundedness principles and BanachSteinhaus theorems with rates. Numer. Funct. Anal. Optim. 3 (1981), 19 - 52.

[3] DICKMEIS, W. and R.J. NeSSEL: Ouantitative Prinzipien gleichmäBiger Beschränktheit und Scharfe von Fehlerabschäzungen. Opladen: Westdeutscher Verlag 1982.

[4] DICKMEIS, W., NESSEL,R.J. and E. van WICKEREN: Quantitative extensions of the uniform boundedness principle. Jahresber. Dt. Math.-Verein. 89 (1987), $105-134$.

[5] NESSEL.R.J. and E. van WICKEREN: On counterexamples in multivariate approximation. In: Approximation in Function Spaces. Proc. Conf. Warsaw 1986 (ed.: Z. Ciesielski). Warsaw: PWN - Polish Sci. Publ. 1989, pp. $269-279$.

[6] POPOV, V.A.: On the one-sided approximation of functions. In: Constructive Function Theory 77. Conf. Blagoevgrad 1977 (eds.: Bl. Sendov and D. Vačv). Sofia: Publ. House Bulg. Acad. Sci. 1980 , pp. $465-468$.

[7] SENDOV, BL. and V.A. POPOV: The Averaged Moduli of Smoothness. Chichester: Wiley 1988 .

\section{Received 03.12.1990}

Dr. Bernhard Buitgenbach, Dipl.-Math. Gerald Lütgens and Prof. Dr. Rolf Joachim Nessel

Rheinisch-Westfälische Technische Hochschule Aachen

Lehrstuhl $\wedge$ für Mathematik

Templergraben 55

D - 5100 Aachen 